MATEC Web of Conferences 21, 12003 (2015)

DOI: $10.1051 /$ matecconf $/ 20152112003$

(C) Owned by the authors, published by EDP Sciences, 2015

\title{
FEM simulation of infeed rotary swaging with structured tools
}

\author{
Marius Herrmann ${ }^{a}$, Christian Schenck, and Bernd Kuhfuss \\ Bime, Bremen Institute for Mechanical Engineering, University of Bremen, 28359 Bremen, Germany
}

\begin{abstract}
Rotary swaging is an incremental cold forming process for rods and tubes. Infeed rotary swaging with structure in the reduction zone of the tools is investigated using a two dimensional finite element simulation. A few geometrical parameters are varied, for cosine and skew stairway shapes. The effective tool angle is kept constant. The influence is evaluated by the radial and axial process forces. Furthermore, the material flow is visualized by the neutral plane. The simulation results are quantitatively compared to each other to analyse the reaction force $\mathrm{F}_{\mathrm{A}}$, which acts against the feeding force. Also, the results serve to find suitable geometries to be transferred to rotary swaging tools for practical application. It is shown that the shapes have a significant effect on the forces and the location of the neutral plane. Finally a first swaging tool is modified with an exemplary geometry for experimental investigations.
\end{abstract}

\section{Introduction}

Rotary swaging has an important field of application in the automotive industry for the production of axes, steering spindles and gear shafts. It features advantages like improved material properties as increased tensile strength, undisturbed fibre flow and for hollow shafts the variable wall thickness for an optimal use of material resources. The diameter of the workpiece is reduced incrementally by the oscillating tool stroke $h_{T}$ (see Fig. 1). The work piece is axially fed in the swaging unit with the feeding force $F_{f}$. Against this force counteracts the axial reaction force $F_{A}$ due to the radial forming force $F_{R I}$ in the reduction zone (I) and the tool angle $\alpha$. To reduce the reaction force conventional tools feature a tungsten carbide layer in the reduction zone to increase the effective friction [1].

In recent years rotary swaging has been studied using finite element method (FEM) to examine strain and stress distributions [2,3] and the material flow by visualization of the neutral plane [4]. In the forming process the neutral plane (NP) represents the plane in which the material features no flow in axial direction. The material before or behind the NP flows against or in feeding direction. The productivity increases with the distance between neutral plane and calibration zone [4]. The investigations are conducted with regard to the influence of axial feed velocity [5] or friction and tool angle [6]. Also, different designs of the reduction zone of the tools are investigated like convex, concave and hybrid contours [7].

\footnotetext{
${ }^{a}$ Corresponding author: herrmann@bime.de
}

This is an Open Access article distributed under the terms of the Creative Commons Attribution License 4.0, which permits unrestricted use, distribution, and reproduction in any medium, provided the original work is properly cited. 


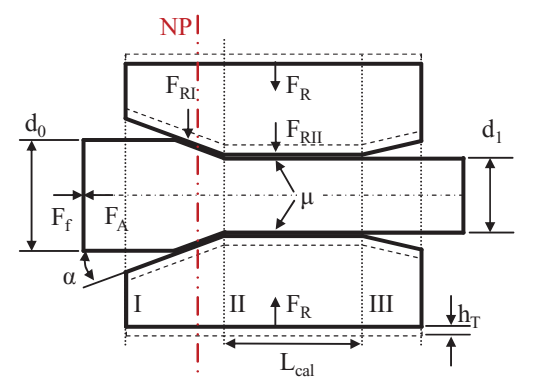

$\begin{array}{llll}\alpha & =\text { tool angle } & \mathrm{h} & =\text { step height } \\ \mathrm{d}_{0} & =\text { initial diameter } & \mathrm{h}_{\mathrm{T}} & =\text { tool stroke } \\ \mathrm{d}_{1} & =\text { final diameter } & \mathrm{l}_{\mathrm{cal}} & =\text { length of zone II } \\ \mathrm{F}_{\mathrm{A}} & =\text { axial reaction force } & \mathrm{NP} & =\text { neutral plane } \\ \mathrm{F}_{\mathrm{f}} & =\text { feed force } & \mathrm{Z}_{\mathrm{NP}} & =\text { distance of the NP } \\ \mathrm{F}_{\mathrm{R}} & =\text { radial force } & \mathrm{I} & =\text { reduction zone } \\ \mathrm{F}_{\mathrm{RI}} & =\text { radial force in zone I } & \text { II } & =\text { calibration zone } \\ \mathrm{F}_{\mathrm{RII}} & =\text { radial force in zone II } & \text { III } & =\text { exit zone }\end{array}$

Figure 1. Nomenclature of infeed rotary swaging.

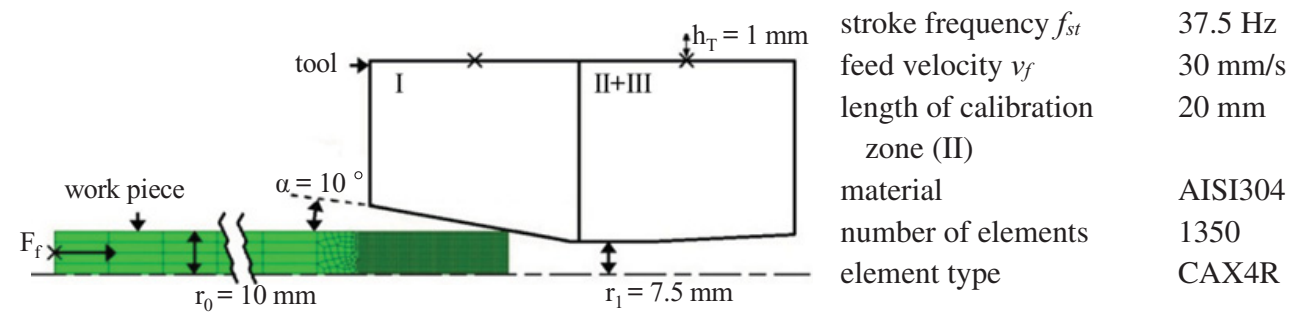

Figure 2. 2D-axisymmetric-model with process parameters for the FE-simulation.

The interest of dry metal forming becomes larger. In case of dry rotary swaging the needed process forces are changing. The distance of the neutral plane $\mathrm{Z}_{\mathrm{NP}}$ is reduced which can lead to a non-economical process $[4,8]$. The approach to cope with this challenge is to control the material flow and thus the required forces by a combination of coating and structuring of the reduction zone of the tools. The coating is on the whole tool with a low friction coefficient to reduce wear and tear as well as cold shuts. The geometry in the reduction zone of the tools in contrast to a plain zone is intended to increase the effective friction. At first, simple contours are investigated by numerical simulations to obtain a basic understanding of the impact on the material flow and the process forces. A qualitative comparison of the simulation results reveals promising structures for the reduction zone.

\section{Modelling}

Two-dimensional axisymmetric simulations are commonly used to simulate radial forging and rotary swaging [2-5, 7]. Due to the shorter computational time compared to three-dimensional simulations more simulations can be calculated what is necessary to study a great variety of different structures. Furthermore, the material flow can be visualized [4]. First investigations with the simulation model which is used for this study are conducted by Moumi [4]. The 2D-axisymmetric model consists of the tool and the work piece and is implemented with the software ABAQUS 6.13-1 (see Fig. 2). The tool is implemented as rigid body. It consists of two parts, the reduction zone (I) and the calibration zone (II). This provides the opportunity to set different friction coefficients between the work piece and the tool. As friction model the penalty formulation and the coulomb friction coefficient is used due to the simplicity and the good results in cold metal forming simulations [9]. The numerical method is based on Abaqus/Explicit due to the fast dynamic process. With an Intel ${ }^{\circledR}$ Core $^{\mathrm{TM}}$ i5-4670 processor the FEMsimulation of one process for one structured tool needs with 4 cores about 4 hours.

After the feeding of the first $35 \mathrm{~mm}$ in the swaging unit the tool is completely filled and a quasisteady state is reached in the process. In consequence, it is not necessary to provide the complete 


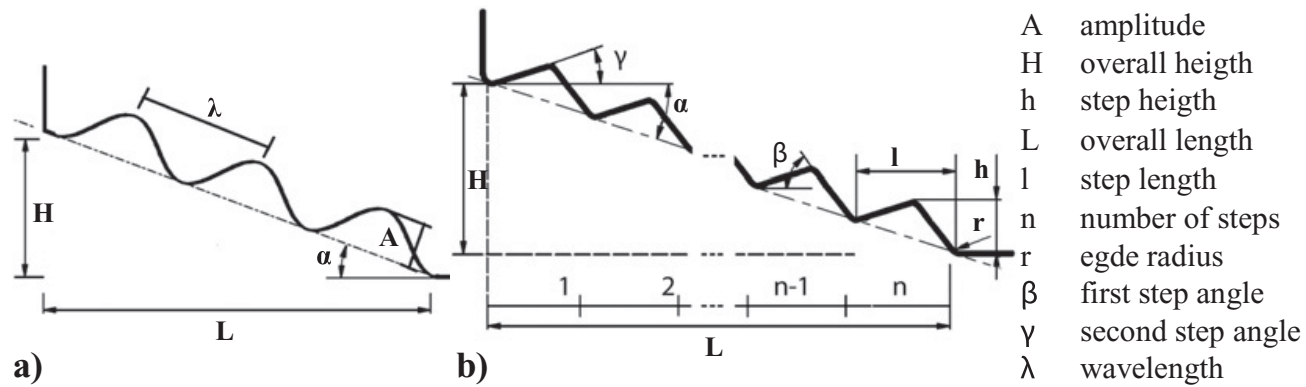

Figure 3. Geometry in the reduction zone of a structured tool; a) cosine, b) skew stairway.

length of the work piece with a fine mesh but only the first $35 \mathrm{~mm}$. The elements are A 4-node bilinear axisymmetric quadrilateral with reduced integration and hourglass control. The mesh size is chosen as large as possible to limit the computation time. Preliminary investigations showed that these restricting constraints do not falsify the results. The workpiece is modelled as an elastic-plastic isotropic material with parameters from literature [10].

The chosen geometries for the structured reduction zone are firstly cosine and secondly skew stairway shapes. The varied parameters of the cosine are the wavelength $\lambda$ and amplitude A (see Fig. 3a); variation: $\lambda=0.9,1.1,1.3 \mathrm{~mm}, \mathrm{~A}=30,40,50,100,150 \mu \mathrm{m}$. To generate asymmetric structures a skew stairway shape is chosen with variable number of steps $\mathrm{n}$ and two step angles $\beta$ and $\gamma$. The skew stairway shape is visualized in Fig. 3b). The selected numbers of steps are between $n=10$ to $n=30$ by an increment of two. The height $\mathrm{h}$ and length 1 are calculated from the fixed overall height $\mathrm{H}=8.28 \mathrm{~mm}$ and overall length $\mathrm{L}=47 \mathrm{~mm}$ leading to $\alpha=10^{\circ}$. Furthermore, the first step angle $\beta=45^{\circ}, 90^{\circ}$ and the second $\gamma=0^{\circ}, 0.1^{\circ}, 1^{\circ}, 2^{\circ}, 5^{\circ}$ are varied. The friction coefficient in the reduction and calibration zone is fixed to $\mu=0.1$ for all simulations derived from the intended coating of the tool.

\section{Simulation results}

With the simulation results the effects of the geometries are analyzed on the radial and axial forces as well as the material flow. The considered axial/radial forces are the average of the maximum axial/radial forces per stroke over the complete quasi-steady state of the process, the radial force is considered for one tool. The results of the comparative simulation without steps are set to $100 \%$. To visualize the material flow one stroke is broken down to multiple time steps. The direction of the material flow is figured white when the flow and the feeding direction coincide and black otherwise (see Fig. 7).

\subsection{Cosine shapes}

The axial and radial forces are represented as a function of the amplitude of the cosine for different wavelengths (see Fig. 4). An effect on both process forces is shown. While the amplitude rises the axial force decreases. Also a taller wavelength leads to a reduction of the axial force. A reduction to $50 \%$ in comparison to the simulation without structured reduction zone is achievable. For the radial force no clear effect of the amplitude is noticeable.

\subsection{Skew stairway shapes}

In Fig. 5 the axial force and in Fig. 6 the radial force is represented as a function of the number of steps for different step angles values. An effect on both process forces is apparent. While the number of steps 

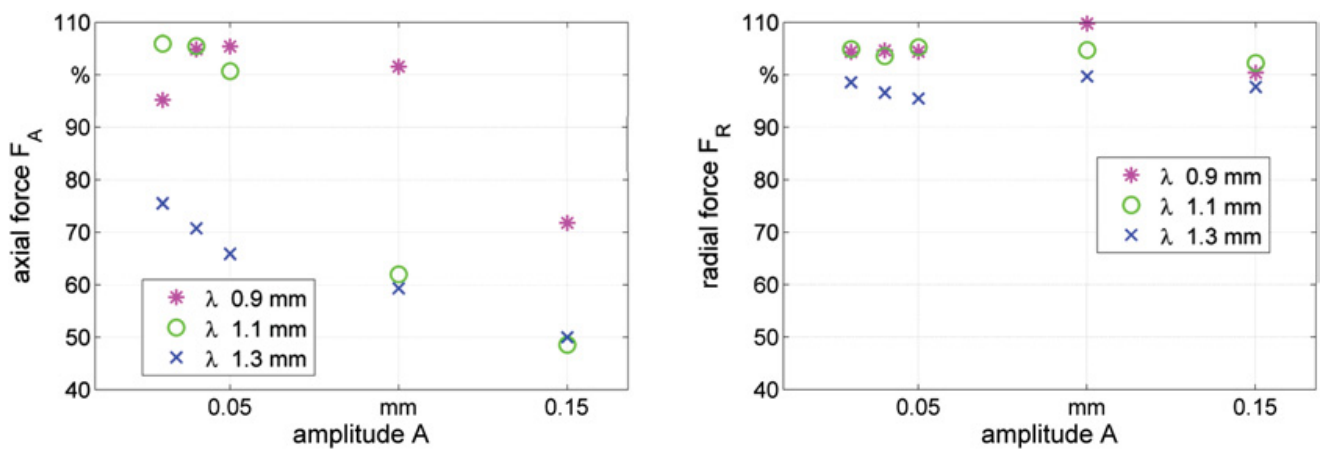

Figure 4. Process forces for the cosine geometry, a) axial force, b) radial force.
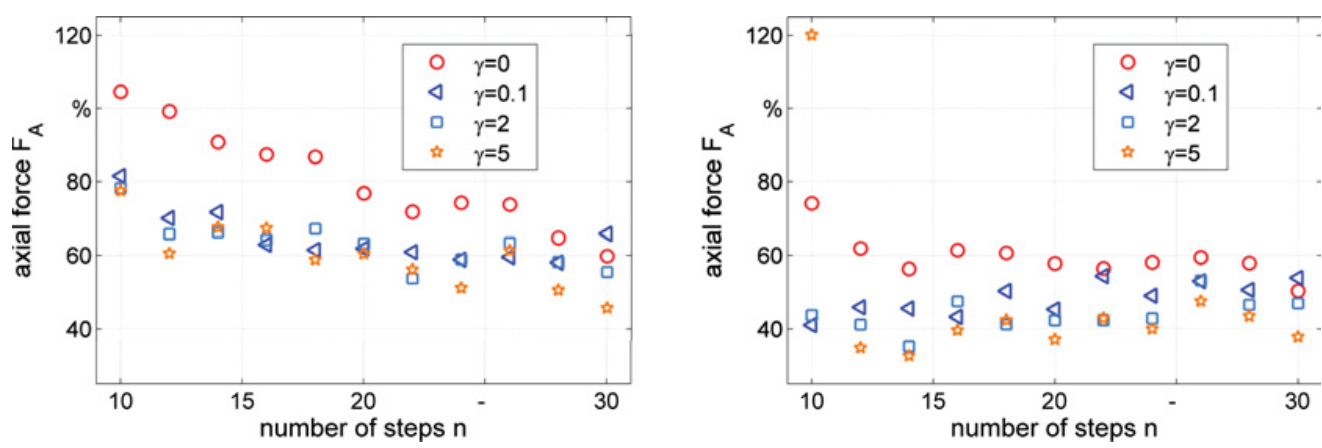

Figure 5. Axial process forces for the skew stairway geometry, a) $\beta=45^{\circ}$, b) $\beta=90^{\circ}$.

decreases the axial force rises. This is especially the case for $\beta=45^{\circ}$ due to the sloped sections (h) of the structure which produce the main part of the axial force. This effect is rarely existing for $\beta=90^{\circ}$. For a higher number of steps the axial force decreases due to the blocking by the sections (1) which are horizontal to the feeding direction. This is favoured for $\gamma>0$.

The radial force is reduced by a smaller number of steps. This is evident in all simulations with different step angles. On the one hand it is based on the fact that with an increasing axial force the radial force decreases. This can be seen in other simulations without structures and different friction coefficients. On the other hand the material flow benefits from the geometry since the flow is less impeded due to the space generated by the skew stairway geometry.

The effect of both step angle $\beta$ and $\gamma$ is illustrated in Fig. 5 and Fig. 6. The axial force is much more influenced by the number of steps with a step angle of $\beta=45^{\circ}$ then with a step angle of $\beta=90^{\circ}$. For equal values of the second step angle $\gamma=0^{\circ}$ and number of steps $\mathrm{n}=20$ with a step angle $\beta=45^{\circ}$ the axial force is $\mathrm{F}_{\mathrm{A}}=76 \%$ whereas with $\beta=90^{\circ}$ the axial force is reduced to $\mathrm{F}_{\mathrm{A}}=57 \%$. This tendency is also shown in the material flow visualized in Fig. 7. By the comparison of Fig. 7a) and Fig. 7b) it is seen that the structure with $\beta=45^{\circ}$ provides the smallest distance of $\mathrm{Z}_{\mathrm{NP}}=30 \%$ between the neutral plane and the calibration zone. This effect is the same for $\gamma=5^{\circ}$, apparent by the comparison of Fig. 7c) and Fig. 7 d) with $\mathrm{Z}_{\mathrm{NP}}=62 \%$ to $\mathrm{Z}_{\mathrm{NP}}=78 \%$.

Also the second step angle $\gamma$ leads to a decrease of the axial force with higher angle value independent from $\beta$ or $\mathrm{n}$. Thus for equal values of $\beta=45^{\circ}$ and $\mathrm{n}=20$ the axial force is decreased from $76 \%$ to $60 \%$ when $\gamma$ is increased from $0^{\circ}$ to $5^{\circ}$. This tendency is again detectable in the 

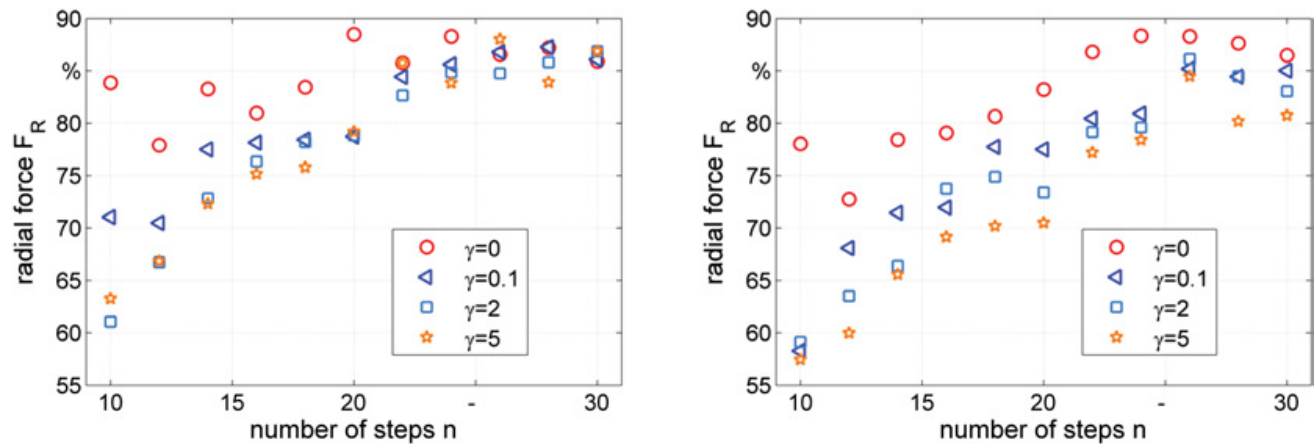

Figure 6. Radial Process forces for the skew stairway geometry, a) $\beta=45^{\circ}$, b) $\beta=90^{\circ}$.
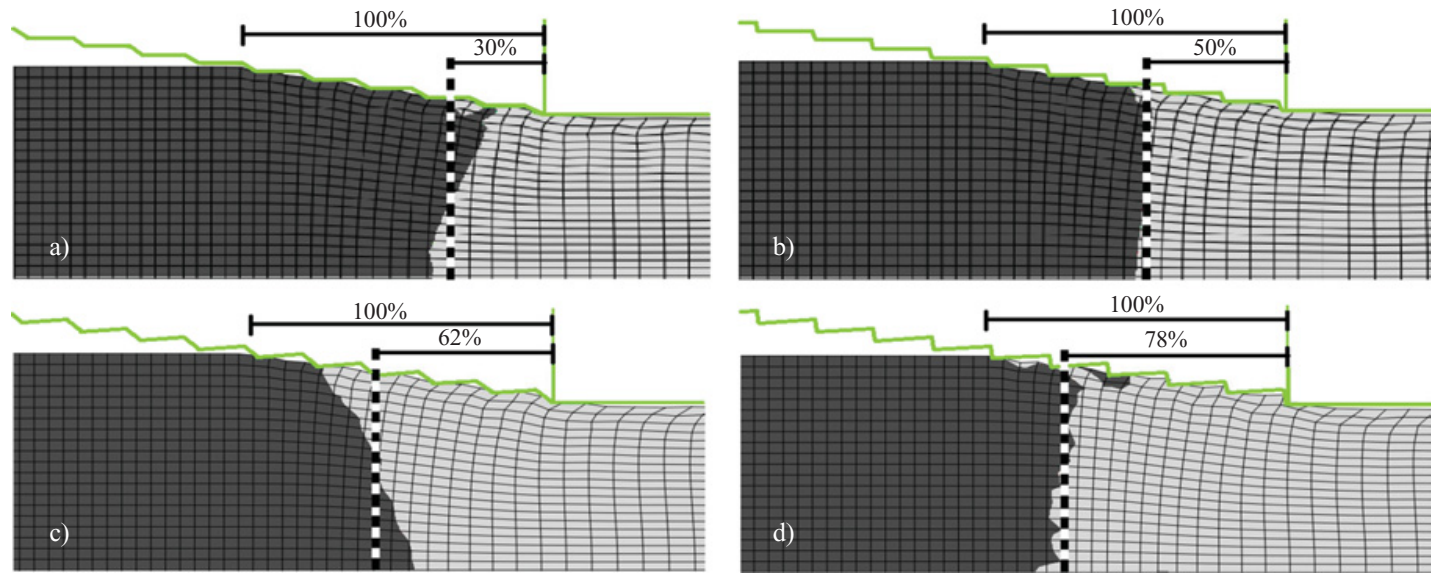

Figure 7. Influence of the Skew stairway shapes with $\mathrm{n}=20$ on the neutral plane, a) $\beta=45^{\circ} / \gamma=0^{\circ}$, b) $\beta=$ $90^{\circ} / \gamma=0^{\circ}$, c) $\beta=45^{\circ} / \gamma=5^{\circ}$, d) $\beta=90^{\circ} / \gamma=5^{\circ}$.

material flow. An increase of the distance between the neutral plane and the calibration zone is shown for a higher value of $\gamma$, see Fig. 7a) compared to Fig. 7c) and Fig. 7b) compared to Fig. 7d).

The radial force is insignificantly influenced by the first step angle $\beta$. For $\beta=90^{\circ}$ the radial forces are smaller. A stronger Influence is generated by the second step angle $\gamma$. For a higher angle value the radial force decreases due to the space generated by the step geometry which leads to a better material flow. For example for equal values of $\beta=9^{\circ}$ and $n=20$ the radial force is decreased from $F_{R}=83 \%$ to $\mathrm{F}_{\mathrm{R}}=70 \%$ when $\gamma$ is increased from $0^{\circ}$ to $5^{\circ}$.

By the comparison of the both chosen shapes in the reduction zone, it is shown that the skew stairway shape has more potential to reduce the process forces, especially for the radial force. However, from the perspective of tool manufacturing these shapes are more difficult to produce.

\section{Experimental testing}

A first experimental implementation of a structured reduction zone for rotary swaging tools is realised. A cosine shape with $\lambda=1.3 \mathrm{~mm}$ and $\mathrm{A}=150 \mu \mathrm{m}$ is chosen. This structure is favoured to manufacture the tools. It is expected that this shape provides a better durability and a higher process stability. 


\section{MATEC Web of Conferences}

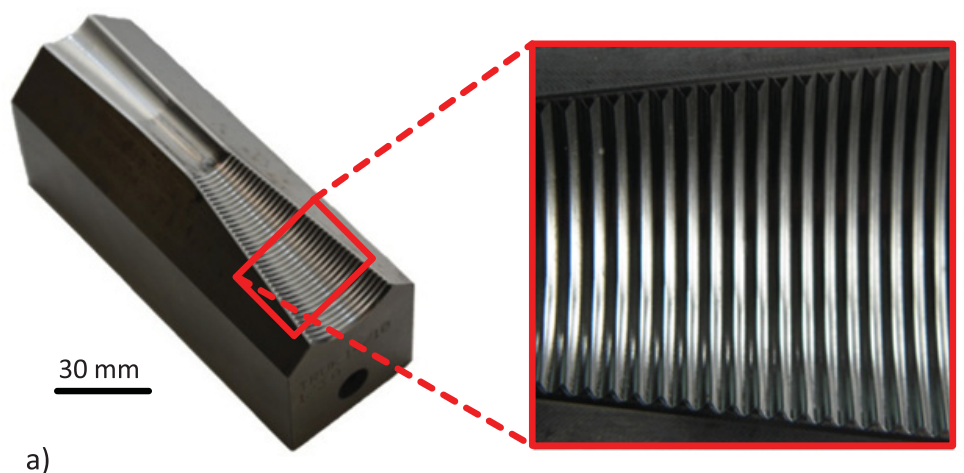

b)
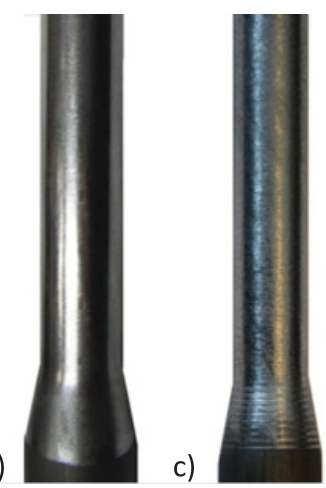

Figure 8. Rotary swaging with cosine structured tool; a) tool $\lambda=1.3 \mathrm{~mm}$ and $\mathrm{A}=150 \mu \mathrm{m}, \mathrm{b}$ ) workpiece formed with conventional tools, c) workpiece formed with structured tools.

The manufactured tool is visualized in Fig. 8a). The rotary swaging of steel tubes (material number 1.0038) was carried out successfully, both under dry and lubricated conditions. The axial reaction force is reduced as evidenced by a lower back pushing of the work piece. However, surface finish of manufactured parts appeared of less quality (higher roughness) compared to rotary swaging carried with conventional tools (compare Fig. 8b) and Fig. 8c)). Further investigations of rotary swaging with structured tools are to be carried out.

\section{Conclusion and outlooks}

The process forces and the material flow are studied during infeed rotary swaging with different shapes with varied geometric parameter values in the reduction zone of the tools. First insights are gained into the effect of geometry in the reduction zone of the tools by the presented simulation model. For the cosine shape the wavelength and amplitude and for the skew stairway geometry the number of steps and two step angles are varied. Following conclusions are drawn: Structured tools can decrease the process forces. Cosine shapes with higher amplitude reduce the axial force while the radial force is rarely influenced. For skew stairway shapes the axial force decreases with a larger number of steps whereas the radial force decreases. With asymmetric shapes a higher force reduction can be achieved especially of the radial force. Also the material flow benefits. With higher values for both step angles both process forces are reduced. With $\beta=90^{\circ}$ a considerable decrease of the axial force is evident as well as for $\gamma$ larger than zero. This effect is also evident in the material flow with the comparison of the distance of the neutral plane to the calibration zone.

In future work a changing structure with variable step height $h$ and step length 1 along the reduction zone will be considered. These studies are promising due to the incremental degree of deformation decreasing in the reduction zone in direction of the calibration zone. Due to the greater effect of the asymmetric skew stairway shape also shifted cosine shapes are of interest. In addition the experimental testing with the presented tools will be carried out to study the effects on geometry and surface quality of the work piece.

The authors gratefully acknowledge the support by the German Research Foundation (DFG, Deutsche Forschungsgemeinschaft) for the sub-project "Potentials of Dry Rotary Swaging" within the priority program 1676 "Dry metal forming - sustainable production through dry processing in metal forming". 


\section{References}

[1] B. Kuhfuss, E. Moumi, "Incremental Forming", 104-113, In: Micro Metal Forming., Ed. F. Vollertsen, Springer Berlin (2013)

[2] A. Ghaeia, M.R. Movahhedya, A. Karimi Taherib, Materials and Design, 29, 867-872 (2008)

[3] O. Pantale, B. Gueye, Journal of Eng., 2013 (2013)

[4] E. Moumi, S. Ishkina, B. Kuhfuss, T. Hochrainer, A. Struss, M. Hunkel, Procedia Eng., 81, 2342$2347(2014)$

[5] L. Rong, Z. NIE, T. ZUO, Trans. Nonferrous Met. Soc. China., 16, 1015-1020 (2006)

[6] A. Ameli, M.R. Movahhedy, Int. J. Adv. Manuf. Technol., 33, 7-17 (2007)

[7] A. Ghaeia, M.R. Movahhedya, A. Karimi Taherib, J. of Mater. Process. Technol., 170, 156-163 (2005)

[8] E. Moumi, B. Kuhfuss, V. Piwek, KIT Scientific Reports, 7591, 161-167 (2011)

[9] X. Tan, Tribology International, 35, 385-393 (2002)

[10] E. Doege, H. Meyer-Nolkemper, I. Saeed, "Fließkurvenatlas metallischer Werkstoffe", Hanser Verlag, Wien (1986)

[11] H. Czichos, K.-H. Habig, J.-P. Celis, "Tribologie-Handbuch: Tribometrie, Tribomaterialien, Tribotechnik", Vieweg + Teubner, Wiesbaden (2010) 